\title{
Towards a better definition of the kilogram
}

\author{
Theodore P Hill ${ }^{1}$, Jack Miller ${ }^{2}$ and Albert C Censullo ${ }^{3}$ \\ ${ }^{1}$ School of Mathematics, Georgia Institute of Technology, 686 Cherry Street, Atlanta, GA 30332, USA \\ ${ }^{2}$ Lawrence Berkeley National Laboratory, 1 Cyclotron Road, Berkeley, CA 94720, USA \\ ${ }^{3}$ Department of Chemistry and Biochemistry, California Polytechnic State University, 1 Grand Avenue, \\ San Luis Obispo, CA 93407, USA
}

Received 15 October 2010, in final form 26 January 2011

Published 1 March 2011

Online at stacks.iop.org/Met/48/83

\begin{abstract}
It is widely accepted that improvement of the current International System of Units (SI) is necessary, and that central to this problem is redefinition of the kilogram. This paper compares the relative advantages of two main proposals for a modern scientific definition of the kilogram: an 'electronic kilogram' based on a fixed value of Planck's constant, and an 'atomic kilogram' based on a fixed value for Avogadro's number. A concrete and straightforward atomic definition of the kilogram is proposed. This definition is argued to be more experimentally neutral than the electronic kilogram, more realizable by school and university laboratories than the electronic kilogram, and more readily comprehensible than the electronic kilogram.
\end{abstract}

\section{Introduction}

The current International System of Units (SI) definition of the kilogram officially recognized by the International Committee for Weights and Measures (CIPM-Comité International des Poids et Mesures) and the General Conference on Weights and Measures (CGPM) is

(D1) The kilogram is the unit of mass; it is equal to the mass of the international prototype of the kilogram.

Even more precisely, it is the mass of that unique International Prototype Kilogram (IPK) immediately after cleaning and washing by a specified method' [1].

This definition (D1) is based on a unique 120-year old platinum-iridium cylinder, and even though there is no incontrovertible proof [2, p 2262], many experts believe that the mass of the IPK is changing in time [12, p 237], based on intercomparisons with other 'identical' kilogram artefacts. For that, and numerous other reasons (e.g. [2, 8, 11, 14]), it is now widely accepted that the kilogram should be redefined in more modern scientific terms. The replacement of the definition (D1) by an intrinsic scientific (non-artefact) definition, based on an 'invariant of nature', has thus been deemed a high priority.

Two recent papers in Metrologia have proposed new definitions of the kilogram and other SI units $[11,12]$. Since the perceived weakness of the current SI definitions of other units such as the ampere, mole and candela 'derives in large part from their dependence on the kilogram...the definition of the kilogram is thus central to the more general problem of improving the SI' [12, p 228]. Accordingly, the Consultative Committee on Units (CCU) and the CIPM have

\section{called for the widest possible publicity to be given to these ideas among the scientific and user communities so that their reactions and views can be taken into account in a timely way... [and] for a wide discussion to take place [12, pp 228-229].}

This paper is a response to that invitation to enter into the discussion about the proposal in [12], and to describe the views and reactions of some scientists and users who are not metrologists. The proposed redefinition of the kilogram will have an impact on all practising scientists and science educators. Our goal is to discuss a recent alternative redefinition of the kilogram $[3,8]$, and to compare the respective definitions.

\section{Proposed definitions}

In [11], the authors describe two possible methods for redefinition of the kilogram: a so-called 'electronic kilogram' based on fixing the value of Planck's constant $h$, and then using a watt-balance method to realize this definition; and an 'atomic kilogram' based on fixing the Avogadro constant 
$N_{\mathrm{A}}$ and then using a silicon-sphere (x-ray crystal diffraction) method to realize the definition.

In a subsequent paper the authors of [12] settle on the electronic kilogram, which is based on two fundamental physics equations: $E=m c^{2}$ and $E=h f$, the first from special relativity theory and the second from quantum mechanics. Using both equations to solve for $m$ yields $m=h f / c^{2}$, and solving for $f$ yields $f=m c^{2} / h$; using the latter they propose three concrete definitions, namely

(D2a) The kilogram is the mass of a body whose equivalent energy is equal to that of a number of photons whose frequencies sum to exactly $\left(299792458^{2} / 66260693\right) \times$ $10^{41}$ hertz.

(D2b) The kilogram is the mass of a body whose de Broglie-Compton frequency is equal to exactly $299792458^{2}$ / $\left(6.6260693 \times 10^{-34}\right)$ hertz.

(D2c) The kilogram, unit of mass, is such that the Planck constant is exactly $6.6260693 \times 10^{-34}$ joule second.

Definitions (D2a) and (D2b) are 'explicit-unit definitions', and (D2c) is an 'explicit-constant definition'; see [12, pp 233234] for details and explanations.

Several justifications for basing the kilogram definition on a fixed value for Planck's constant were identified in [12]. One reason was that the definition would 'lead to a simplification ... for all precise electrical measurements'. According to researchers at the All-Russia Research Institute for the Metrological Science (VNIIMS), however, 'using the Watt balance with subsequent introduction of an electrical kilogram... will actually require the creation of a quantummechanical current standard' [8, $\mathrm{p}$ 587]. A second reason listed in [12] was 'from the point of view of fundamental physics, Planck's constant plays a more important role than Avogadro's number'. While many physicists may agree with that opinion, most chemists would not. The authors of [12] also propose redefinitions of other SI units, but we will restrict attention to the redefinition of the kilogram.

Our own proposed redefinition of the kilogram is a simple, concrete version of the method of fixing the Avogadro constant $N_{\mathrm{A}}(\mathrm{cf}[3,8])$, namely

(D3) A kilogram is the mass of $84446889^{3} \times 1000 / 12$ unbound atoms of carbon-12 at rest and in their ground state.

Note that this latter definition bases the kilogram on the invariant mass of a carbon-12 atom, satisfying one of the early requirements for redefinition, and that the number of carbon- 12 atoms specified in (D3) is an integer, which is 'entirely natural and should be satisfied' [8, p 588]. Other choices for the exact numerical value of the number of carbon-12 atoms in a kilogram are also possible, such as one which specifies an exact number of atoms in a gram (see section 3), one that takes into account the graphite or diamond lattice structure of carbon-12 [3, 8], or one that is a decimal approximation to eight or nine orders of magnitude of the mean of the latest recommended value. Definition (D3) was chosen to make the mole and the Avogadro constant particularly simple, and because it is very close to the current recommended values (see section 4). The exact numerical value chosen for (D3) is quite flexible; for instance the proposal in [11, p 75] states 'we believe that even if it were to be eventually discovered that the value of $h$ or $N_{\mathrm{A}}$ chosen to redefine the kilogram were such that [the relative deviation in mass of the new kilogram from the mass of $I P K] \approx 10^{-6} \ldots$ the consequences could be better dealt with through a redefinition now.'

As explained clearly in [6], from the equation relating Planck's constant, the fine structure constant, the Rydberg constant, the speed of light and the electron mass, it is possible to fix exactly any two of the three fundamental constants Avogadro's number, Planck's constant and the carbon-12 molar mass. (For arguments supporting fixing the carbon-12 molar mass, see [6].)

\section{Practical realizations}

Once a new definition of the kilogram such as (D2a-b-c) or (D3) is adopted, no man-made object (including the IPK) will ever have mass exactly one kilogram, except by pure chance and then only instantaneously. However, there still will be a need for practical realizations of the kilogram similar to the various national copies of the IPK. Initially, the existing prototype copies will serve that role, but as time goes on, more accurate copies will certainly be needed, and perhaps more countries will want copies. In this section, we will review some of the issues in actually realizing prototype kilograms under the new definition, in other words, constructing or calibrating scales that are highly accurate.

The long-term goal is to design

a comparatively easy-to-use apparatus that can enable the experimental realization of the new definition of the kilogram with the appropriate uncertainty at any place at any time by anyone [12, p 238].

That is exactly what happened in 1983 when the metre was redefined as the distance light travels in exactly $1 / 299792458 \mathrm{~s}$, thereby eliminating the need for the official artefact platinum-iridium metre stick forever (see [1] for a history of earlier definitions). This is an 'explicit unit' type of definition. An alternative (and equivalent) 'explicit constant' definition would be: the metre is that length such that the speed of light is exactly $299792458 \mathrm{~m} \mathrm{~s}^{-1}$. Since that 1983 redefinition, any student with a stopwatch, laser pointer, strobe light and rotating mirror can construct a reasonably accurate 'metre stick' independent of any other prototype.

Definitions (D2a-b-c). Although definitions (D2a-b-c) are attractive from the standpoint of theoretical physics, it has been argued that 'the basis of this definition will not be a natural invariant, such as the mass of a carbon atom, but an artificially created electromechanical device, the Watt balance, with a large number of sources of systematic uncertainty' [8, p 590].

Moreover, the watt-balance method requires substantial resources, hence their rare status. The one at the National Institute of Standards and Technology (NIST) is two stories high, cost over US $\$ 1.5$ million to set up, and requires a team 
of between three and five expert scientists working on the project at any one time, as well as considerable use of expensive liquid helium for the two superconducting magnets. According to the head of the NIST watt-balance unit, the problem with the watt-balance method (as well as the silicon lattice sphere method) is that the underlying experiment is by nature very sensitive. There are not many scientists with the expertise to build and run a watt-balance, and not many countries are willing to afford such expenses year after year. In fact, the National Physical Laboratory in the UK, the site of the first watt-balance, recently ceased funding of its own watt-balance experiments, dismantled the device, and shipped it to Canada.

The problem of coping with the various sources of error in the watt-balance experiment is compounded dramatically by the magnitudes specified in the definitions. Definitions (D2a-b) entail a de Broglie-Compton frequency in hertz (cycles per second) of more than $10^{50} \mathrm{~Hz}$. Since the caesium (caesium-133) clock frequency to determine the second is only $9192631770 \mathrm{~Hz}$, or about $9 \times 10^{9} \mathrm{~Hz}$, the defining frequency in $(\mathrm{D} 2 \mathrm{a}-\mathrm{b})$ is 41 orders of magnitude greater than that of the number of vibrations defining the second. Moreover, the proposed exact constant in (D2a) and (D2b) is not only larger than $10^{50}$, but also is a non-terminating (infinite) decimal, which is still only an approximation even when rounded to a billion digits. In [10, p 2] the authors maintain that 'It would be inconvenient to quote concentrations... with numbers of the order $10^{23} \ldots$, but definitions (D2a) and (D2b) even require estimates of frequencies 27 orders of magnitude greater than that.

We are not aware of any proposed simple laboratory experiments that students and university professors can use to construct a rough approximation of a kilogram mass based on definitions (D2a-b-c), unlike the redefinition of the metre that permitted a standard school laboratory experiment to construct a rough metre stick.

Definition (D3). One indirect method of practical realization of (D3), of course, is to use a watt-balance experiment and the various physics equations relating the fundamental constants. Although data analysis of the experiments underlying the wattbalance and the crystal x-ray diffraction methods uncovered two major discrepancies [12,13], and the fundamental problem of how to combine data from different types of independent experiments is indeed a difficult one, recent advances in statistical theory may well prove very useful ( $\operatorname{cf}[4,5])$, and for practical realizations, either experimental method may be used to generate artefacts based on either an electronic or atomic kilogram definition.

The most direct practical realization of (D3) is currently available through several laboratories in the Avogadro Project, where silvery softball-sized artefacts of single-crystal silicon spheres of high purity and nearly spherical shape yield an estimate of the number of silicon-28 atoms in a given macroscopic mass. The first such results were published in 2004, and currently only two such spheres exist, each costing about $\$ 3.2$ million and manufactured and maintained by master opticians. The objective of this method is simply to estimate the number of atoms in the sphere, using estimates of the imperfect purity of the silicon isotopes, the average volume of a silicon-28 atom, and the radius of the rough sphere. Thus the sphere itself is a sophisticated (and necessarily imperfect) realization of any atom-counting definition such as in (D3).

In contrast to definitions (D2a-b-c), definition (D3) also allows a direct and simple rough prototype of a kilogram mass to be constructed in a school laboratory, or even at home: a block of nearly pure carbon, cut so that it is roughly $8.11 \mathrm{~cm}$ (or as close to 368855762 carbon-12 atoms as possible) on a side, will be approximately one kilogram. Of course, the exact dimensions depend on the form of carbon used-graphite, say, or diamond — and on its crystal lattice structure (cf $[3,8]$ ) as well as on mass differentials to compensate for binding energies, since the atoms in the lattice will not be free atoms. But it should be emphasized that definition (D3) does not specify a method of realization, and although at this point in time it is not yet possible to obtain exact counts of individual atoms, even when they are in a crystal lattice, that is merely a question of time. (For additional information about differences between the electronic and atomic kilogram definitions, see also the section 'Comparison of the Two Basic Methods for Redefinition of the Unit of Mass Associated with the Avogadro and Planck Constants' in [8]).

\section{Educational aspects}

One of the most crucial considerations of any redefinition of the kilogram and other SI units is the legacy we leave to the next generation of scholars. As [12, p 228] declares

\section{since it is important that the basis of our measurement system be taught in schools and universities, it is preferable, as far as modern science permits, that the definitions of base units be comprehensible to students in all disciplines.}

Definitions (D2a-b-c). To understand these three proposed redefinitions of the kilogram requires knowledge of physics at the advanced university or even graduate level, including special relativity and quantum mechanics. In response to our multiple queries for a concise definition of the kilogram which would be suitable for inclusion in an introductory-level science textbook, we did not receive any replies which addressed these pedagogical concerns.

We feel that when a redefinition of the kilogram is eventually adopted, that definition, as [12] said, should be made 'comprehensible to students in all disciplines' as far as modern science permits, and the present wording of $(\mathrm{D} 2 \mathrm{a}-\mathrm{b}-\mathrm{c})$ does not seem to meet that goal.

Definition (D3). Modern science does permit a simple redefinition of the kilogram that is easily comprehensible to students in all disciplines, and (D3) is one such example. Students need only have an idea what an atom of carbon-12 is. Moreover, the great advantage of definition (D3) is that it also allows clean and concise definitions of the Avogadro constant $N_{\mathrm{A}}$ and the mole [8-10], namely

(D4). Avogadro's constant, the number of atoms in 12 grams of carbon-12, is $N_{\mathrm{A}}=84446889^{3}$, 
and

(D5). The mole is the amount of substance that contains exactly $84446889^{3}$ specified elementary entities, which may be atoms, molecules, ions, electrons, other particles or specified groups of such particles.

The choice of a perfect numerical cube for the constant defining $N_{\mathrm{A}}$ and the mole was motivated by the simple mathematical fact that an open region in space has positive volume if and only if it contains a perfect geometrical cube. Hence a mole of any atom or molecule, for example, always contains a perfect geometrical cube, and the numerical cube chosen is simply the largest possible geometrical cube a mole can contain. The magnitude of the defining side length of the cube is of the order $10^{8}$, which is consistent with current goals of metrology. Moreover, a cube is easy to visualize, and this is of great value in making the definition comprehensible to students in all disciplines. Additional reasons for choice of a perfect cube, in addition to its elegance and simplicity, are outlined in [3].

A simple, clean definition of the mole is especially important in chemistry (e.g., see [6,9]). The Chair of the Committee on Nomenclature, Terminology and Symbols of the American Chemical Society wrote that by simply fixing an integral value for Avogadro's number in this manner, 'much of what seems to confuse many students about the mole in introductory courses will be dampened' [7]. And, as confirmed in an earlier paper by the same proposers of definitions (D2a-b-c), an atom-counting definition of the kilogram that fixes $N_{\mathrm{A}}$ (as (D3) and (D4) do) 'is simple, conceptually, enabling it to be widely understood... [and] allows the mole to be redefined in a simpler and more understandable way' [11, p 77].

One of the official recommendations of the CIPM was to 'further encourage National Metrology Institutes to pursue national funding to support continued relevant research' [12, p 245]. But in making a decision to discard an easilyunderstood atom-counting definition in favour of a wattbalance definition (D2a-b-c), even the appearance of ulterior funding considerations should be carefully avoided.

\section{Conclusions}

In our opinion, the proposed new definition of the kilogram (D3) is (i) more experimentally neutral than definitions (D2a-b-c), which heavily favour the sensitive watt-balance experiments; (ii) much easier for school and university science laboratories to use to make rough direct realizations than (D2ab-c); and, of utmost importance to future generations who will use the SI, (iii) vastly easier to comprehend and visualize than (D2a-b-c).

Although the President of the $\mathrm{CCU}$ has insisted that 'the time [for redefinition of the kilogram] is not only right, but urgent', the former Director of the Mass Department at BIPM is cautious about the 2011 deadline: 'It's not yet urgent. ... people have been living with this for years' and it is this view that we share. But if a decision is to be made, we strongly prefer the elegant redefinition (D3). For additional arguments supporting a preference for an atomic kilogram definition over an electronic kilogram definition, see [8] and the references therein.

At its September 2010 meeting the Consultative Committee for Units recommended: 'However, the CCU was firmly of the opinion that it is now time to declare to the wider scientific and user public exactly what is likely to be proposed, so that it can be properly and openly discussed.' We agree with their conclusion that this topic warrants timely and broad discussion with stakeholders.

\section{Acknowledgments}

The authors are grateful to Peter Becker, Richard Davis, Ronald Fox, Paul Karol, Peter Mohr, Richard Steiner, Barry Taylor, Metrologia Editor Janet Miles and two referees for helpful communications and suggestions. The first author also wishes to thank Peter Rusch for his kind invitation to attend the session of the Committee on Nomenclature, Terminology and Symbols at the 2010 National Meeting of the American Chemical Society in Boston.

\section{References}

[1] Bureau International des Poids et Mesures 2006 SI Brochure 8th edn http://www.bipm.org/utils/common/pdf/ si_brochure_8.pdf

[2] Davis R 2005 Possible new definitions of the kilogram Phil. Trans. R. Soc. A 363 2249-64

[3] Fox R F and Hill T 2007 An exact value for Avogadro's number Am. Sci. 95 104-7

[4] Hill T 2010 Conflations of probability distributions Trans. Am. Math. Soc. at press http://arxiv.org/abs/0808.1808

[5] Hill T, Miller J and Fox R F 2010 How to combine independent data sets for the same quantity http://arxiv.org/abs/1005.4978

[6] Jeannin Y 2010 What is a mole?: old concepts and new (continued) Chem. Int. 32 (1) 8-11

[7] Karol P 2007 Am. Sci. 95291 (Letter to the Editors)

[8] Khruschov A 2010 Fundamental problems in metrology; possible definition of the unit of mass and fixed values of the fundamental physical constants Meas. Tech. 53 583-91

[9] Lorimer J 2010 What is a mole?: old concepts and new Chem. Int. 32 (1) 6-7

[10] Mills I and Milton M 2009 Amount of substance and the mole Chem. Int. 31 (2) 3-7

[11] Mills I, Mohr P, Quinn T, Taylor B and Williams E 2005 Redefinition of the kilogram: a decision whose time has come Metrologia 42 71-80

[12] Mills I, Mohr P, Quinn T, Taylor B and Williams E 2006 Redefinition of the kilogram, ampere, kelvin and mole: a proposed approach to implementing CIPM recommendation 1 (CI-2005) Metrologia 43 227-46

[13] Mohr P, Taylor B and Newell D 2007 The fundamental physical constants Phys. Today 60 (7) 52-5

[14] Robinson I 2006 Weighty matters Sci. Am. 29 102-9 